\title{
Mental Health and Quality \& Safety of Care in Czech Residential Institutions during the COVID-19 Pandemic: A Mixed-Methods Study
}

\section{Zoe Guerrero $^{1}$ - Akmal-Alikhan Aliev ${ }^{1}$ - Lucie Kondrátová ${ }^{1}$ - Bibiána Jozefiaková ${ }^{1,2}$. Natálie Nesázalová ${ }^{1}$ · Josephine Gabriela Saňáková ${ }^{1}$ P Petr Winkler ${ }^{1,3}$}

Accepted: 14 March 2021 / Published online: 5 April 2021

(c) The Author(s), under exclusive licence to Springer Science+Business Media, LLC, part of Springer Nature 2021

\begin{abstract}
Long-stay institutions have been considerably affected by the COVID-19 pandemic. We aimed to assess the mental health of clients and staff as well as quality and safety of care in long-stay institutions during the state-of-emergency in the Czech Republic in response to COVID-19 pandemic. We found a high prevalence of poor mental health outcomes in clients (46\% poor well-being, 58\% depression, $45 \%$ anxiety) and staff (17\% poor well-being, 22\% depression, $14 \%$ anxiety). In clients, COVID-19 health-related and economic worries were associated with depression $(1.79,95 \% \mathrm{CI}=1.14 ; 2.8$ and $2.28,95 \% \mathrm{CI}=1.27 ; 4.08$ respectively) and anxiety $(1.63,95 \% \mathrm{CI}=1.11 ; 2.4$ and 1.85 , $95 \% \mathrm{CI}=1.2 ; 2.85$ respectively) and in staff with any mental health outcome $(1.92,95 \%$ $\mathrm{CI}=1.33 ; 2.77$ and $1.75,95 \% \mathrm{CI}=1.15 ; 2.66$ respectively). Lack of information and communication from authorities, lack of protective equipment and logistic difficulties were reported as challenges. Delivery of care was mostly disrupted as well as admission and discharge processes. Other reported issues included lack of staff, lack of activities for patients or an increase in usage of restrictive measures. Best practices and key future measures were identified by each institution, a summary of which is presented in the article.
\end{abstract}

Keywords Covid-19 · Mental health · Long-stay institution · Residential institution $\cdot$ Czech Republic

Zoe Guerrero and Akmal-Alikhan Aliev contributed equally to this work.

Akmal-Alikhan Aliev

Akmal.aliev@hotmail.com

1 Department of Public Mental Health, National Institute of Mental Health, Topolová 748, 25067 Klecany, Czech Republic

2 Social Health Institute, Palacky University, Univerzitní 22, 77111 Olomouc, Czech Republic

3 Institute of Psychiatry, Psychology \& Neuroscience (IoPPN), King's College London, 16 De Crespigny Park, London SE5 8AF, UK 


\section{Introduction}

Long-stay institutions have been considerably affected by the current COVID-19 pandemic. They have been at a higher risk of outbreaks because of factors such as limited screening capacities, lack of personal protective equipment, limited space for isolation of COVID-19 positive residents, difficulties in acceptance of changes in routines from both clients and staff, and decreased cognitive capacities and unfavourable health habits of some of the residents. [1-4] In addition, residential care staff are typically not trained for dealing with infectious diseases and they often lack the skills and education to do so. [5]

Mental health and wellbeing of both staff and clients of long-stay institutions might have been negatively affected as well. While the possible decreased economic stability of residential institutions might have had an impact on the mental health of the staff, and lack of group activities such as group therapy might have had an impact on the mental health of the clients, social isolation from the general population and newly arisen difficulties in care provision might have negatively affected both groups. [3,4]

Opinions on how to increase the quality and safety of care in long-stay institutions, especially in psychiatric hospitals, during the COVID-19 pandemic emerged instantly. Du \& OuYang [6] and Hsu et al. [5] emphasized a need for appropriate, up-to-date and well-communicated guidelines, especially in larger residential institutions. Thome et al. [7] suggested a need to avoid violation of human rights of inpatients and proposed creating a new set of guidelines regarding coercion. D'Agostino et al. [8] pointed to the need to set up functioning pathways for discharged patients to secure continuity of care. Chevance et al. [9] suggested that online tools could be utilized in that regard, and Kopelovich et al. [10] pointed out that educating discharged patients about basic techniques for mental health self-care could prevent anxiety. Achieving a continuous provision of quality care even amidst a crisis might be difficult, but simple logistics such as availability of specialist staff, for example, psychologists/psychiatrists $[4,11]$, helps to prevent future relapses, which is especially important since residential institutions might face a possible increase in demand of care and larger waiting lists in the near future. This may be the case, firstly, because people with pre-existing mental disorders report worse outcomes during lockdowns and secondly, because of an increase of poor mental health outcomes in the general population. [12-14]

While the above-mentioned suggestions are well-founded, to our knowledge, the provision of care in residential institutions during the COVID-19 pandemic has been systematically analysed only within the World Health Organization (WHO) rapid assessment of quality and safety of care in long-term residential institutions for people with mental health problems across Europe [15]. The findings from this study show that delivery of care proved challenging during the pandemic, mostly due to a lack of guidelines and communication between key stakeholders. Recommendations stemming from the report include implementing plans at the institutional level in case of infection and revising admissions protocols, as well as maintaining care which is of acceptable quality. However, this study did not take into account residential institutions other than psychiatric hospitals and did not assess mental health among staff or clients.

In the Czech Republic, the nationwide state-of-emergency in response to COVID-19 lasted from March 12th to May 17th highly impacting the work of long-stay institutions. We employed mixed-method design to make an in-depth assessment of the impact of the pandemic and the enhanced restrictive measures during this time. Firstly, we aimed to quantitatively assess the mental health of both staff and clients of the institutions. Secondly, we used qualitative design to get detailed insights into COVID-19 related challenges, 
lessons learned, and best practices in care provision and prepare recommendations and interventions to improve preparedness for future outbreaks.

\section{Methods}

\section{Participants}

We considered all types of long-term residential care providers in the Czech Republic. The institutions belonged to three ministries as follows; Ministry of Youth, Education and Sport (MYES): residential treatment centres, foster homes and child diagnostic institutes. Ministry of Labour and Social Affairs (MLSA): elderly homes, nursing homes and homes with special arrangements. Ministry of Health (MH): psychiatric hospitals. Definitions of types of institutions within the Czech context can be found in the Online Resource.

We asked delegates of respective ministries, who participated in the Working Group on COVID-19 Pandemic \& Mental Health established under the Governmental Council for Mental Health in the Czech Republic, to provide a convenient but heterogeneous sample of institutions able to participate in the study. Both institutions that have had COVID-19 positive cases and those that have had no such cases were included in the sample.

Directors of all institutions were contacted by PW, they were informed about the study and asked to participate. Institutions with a capacity larger than 50 beds were asked to include one ward only. Directors were informed about both questionnaire survey and focus groups, and they were asked to be of assistance in planning and conducting the one-day visits in their institutions. Specifically, they were asked for enabling distribution and collection of questionnaires, providing a room for focus groups, and facilitating contact between interviewers and both staff and clients. Special arrangements were agreed with institutions belonging to the MYES where it was necessary to get informed consent from parents or legal representatives of clients younger than 18 years.

We conducted an online training of 39 evaluators who subsequently, between April 5th and May 25th, visited the residential institutions. During their 1-day visits, in each institution, the evaluators distributed a questionnaire assessing the mental health of staff and clients, and conducted three focus groups (with management, regular staff, and clients of an institution) focused on quality and safety of care during the COVID-19 pandemic.

\section{Data and Measures}

Alongside obtaining socio-demographic data including age, sex, marital status and level of education, we used three scales: General Anxiety Disorder-7 (GAD-7), Patient Health Questionnaire-9 (PHQ-9), and World Health Organization Well-Being Index (WHO-5) to screen for depression and anxiety symptoms and poor well-being. All three scales are widely used in the Czech Republic. These tools are aimed at screening mood disorders and provide only probable diagnosis, which should be confirmed by further evaluation.

GAD-7 is a seven-item scale, which comprises questions on anxiety symptoms experienced over the past two weeks before the survey. Items are coded on an ordinal scale 
(0-3). Symptoms, which the respondent experienced nearly every day score 3 points; 0 points reflect a response where the respondent did not experience a symptom at all. The total score is calculated by adding the points obtained for each of the 7 items. Higher total scores correspond to greater severity of anxiety. [16]

PHQ-9 is a tool that comprises 9 questions on the presence and severity of depressive symptoms experienced over the past two weeks before the survey. The structure of the tool and calculation of scores is similar to GAD-7. The higher the total score the more severe is depression. [17]

WHO-5 is a short and generic tool to measure subjective well-being based on five positively phrased statements. Respondents are asked to rate each statement on a scale from 0 to 5. The overall score ranges from 0 (absence of well-being) to 25 (maximal well-being). [18]

Additionally, we included questions related to COVID-19 health and economic worries among the respondents. We assessed these worries (direct and indirect), expressed as the number of items with strong worries about the impact of the pandemic on health and financial situation (minimum 0, maximum 2). The precise wording of COVID-19 related questions and distribution of responses are provided in the Online Resource. The whole questionnaire consisted of 31 questions and was used among clients, staff and managers of the institutions.

For qualitative data collection, all respondents were divided into groups based on their positions in an institution (client-staff-managers). Each focus group discussions consisted of two parts: structured questioning and open questioning.

For structured questioning, at the beginning of each focus group participants were asked to reach a consensus on questions that were relevant to the predefined themes (Information and Communication, Implementation of Measures, Delivery of Care, Quality of Experience). These themes were derived from the WHO qualitative study of quality of care in long-term mental health care institutions across Europe during the pandemic [15]. For a full list of questions for both staff and client groups see Table 2 in the Online Resource. If there was a consensus in the group, the moderator recorded the answer in a form; if there was not a consensus in the group, no answer was collected (therefore producing missing data).

Open questions related to the above-mentioned core themes were asked by the moderator of the session and notes were taken while the respondents were answering. Each of the 4 themes was sub-divided into three areas: problems and challenges, solutions and future needs.

\section{Quantitative Analysis}

Quantitative analysis was conducted using Stata software version 16. First, we derived descriptive statistics of socio-demographic variables and prevalence rates of anxiety, depression and poor well-being stratified by sex among clients, staff and managers as well as by types of institutions. Additionally, we provide prevalences for a sample of clients stratified by three main age groups (children, adults and elderly) excluding psychiatric hospitals since mental health problems are the primary reasons for hospitalization in these facilities. Severity levels of depression and anxiety are given too, and prevalence of moderate and severe outcomes are combined to compare them with the results of the mental health survey conducted on the Czech general population during the pandemic [19]. 
We used logistic regression to identify if strong COVID-19 health and economic worries are associated with the presence of poor well-being (1), depression (2), anxiety (3) or any of the outcomes (4). We controlled for age, sex, level of education, marital status and type of institution and report results as odds ratios (OR) with $95 \% \mathrm{CI}$, considering associations with $\mathrm{p}<0.05$ as statistically significant. We applied pair-wise deletion to missing data due to univariate pattern of missing (ie same participants had missing data on several items in the questionnaire).

\section{Focus Groups Analysis}

Structured questioning data were tabulated using Stata software version 16. Following tabulation, data were analysed for discrepancies in responses between each participant group (staff, managers, clients). In terms of the open questioning, three researchers (ZG, NN, LK) analysed these data using an open coding method [20]. A code was assigned to each answer. We did not use a predefined set of codes, as codes were generated continuously. Following this stage, codes were grouped in clusters and the frequency of occurrence for each cluster was calculated. Institutions were divided into child and adult facilities for all stages of qualitative analysis.

\section{Results}

\section{Participants}

Twenty-seven institutions across the Czech Republic participated in the survey. Not all institutions that were initially approached participated in the survey, because of logistic reasons and time constraints. Most of the institutions $(n=12)$ were under the responsibility of the MLSA followed by the MYSE $(n=10)$ and the MH $(n=5)$.

In each institution three respondent groups were surveyed, where possible: clients, staff and management of the institution. When parents' informed consent was needed for child participants to take part in the survey, they were excluded from the study. This was the case in four child institutions. Staff in three institutions and management in four institutions did not participate in the survey because of logistic reasons and time constraints. In total 378 clients, 443 staff and 49 managers participated in the study (Table 1).

Table 1 Number of participants by type of institution

\begin{tabular}{llll}
\hline Type of institutions & Clients, $\mathrm{n}$ & Staff, $\mathrm{n}$ & Managers, $\mathrm{n}$ \\
\hline Psychiatric hospitals & 103 & 58 & 4 \\
Elderly homes & 86 & 24 & 6 \\
Nursing homes & 38 & 159 & 9 \\
Homes with special arrangements & 36 & 52 & 12 \\
Residential treatment centres & 57 & 72 & 8 \\
Child diagnostic institutes & 15 & 28 & 5 \\
Foster homes & 43 & 50 & 5 \\
Total & 378 & 443 & 49 \\
\hline
\end{tabular}


For further quantitative analysis staff and manager groups, due to the small sample of the latter, were combined into one 'staff' group. Full socio-demographic details are given in Table 2. Females represented 55\% of the participants among clients and $78 \%$ among staff. Mean age of clients was 45.6 and the 12-20 age group was represented by nearly a third of the participants followed by the $71+$ age group (24\%). Mean age of staff was 45.5 with most of the participants of the age of 41-50. Half of all clients reported elementary education and $60 \%$ being single. More clients (13\%) expressed strong worries regarding the health consequences of COVID-19 than staff (7\%). Additionally, $8 \%$ of clients and 5\% of staff reported strong worries about the economic consequences of COVID-19 pandemic.

\section{Mental Health of Participants}

Binary mental health outcomes among the two respondent groups are presented in Table 3 . Nearly half of all clients met diagnostic criteria for anxiety or poor well-being and nearly $60 \%$ for depression. About a fifth of staff members reported depressive symptoms; $17 \%$ and $14 \%$ fulfilled criteria for poor well-being and anxiety respectively.

Table 2 Sociodemographic characteristics of the participant

\begin{tabular}{|c|c|c|c|}
\hline & & Clients & Staff \\
\hline \multirow[t]{8}{*}{ Age } & Mean (SD) & $45.6(27.5)$ & $45.5(10.7)$ \\
\hline & $<20$ years & $119(31.9 \%)$ & $3(0.6 \%)$ \\
\hline & 21-30 years & $30(8.0 \%)$ & $56(11.5 \%)$ \\
\hline & $31-40$ years & $40(10.7 \%)$ & $80(16.4 \%)$ \\
\hline & $41-50$ years & $29(7.8 \%)$ & $185(37.8 \%)$ \\
\hline & $51-60$ years & $31(8.3 \%)$ & $136(27.8 \%)$ \\
\hline & $61-70$ years & $35(9.4 \%)$ & $27(5.5 \%)$ \\
\hline & $70+$ years & $89(23.9 \%)$ & $2(0.4 \%)$ \\
\hline \multirow[t]{2}{*}{ Sex } & Male & $168(44.8 \%)$ & $107(21.8 \%)$ \\
\hline & Female & $207(55.2 \%)$ & $385(78.2 \%)$ \\
\hline \multirow[t]{5}{*}{ Education } & Elementary school & $171(50.4 \%)$ & $12(2.4 \%)$ \\
\hline & Secondary school & $82(24.2 \%)$ & $109(22.3 \%)$ \\
\hline & High school & $59(17.4 \%)$ & $166(34.0 \%)$ \\
\hline & Bachelor degree & $10(3.0 \%)$ & $74(15.1 \%)$ \\
\hline & Master degree & $17(5.0 \%)$ & $128(26.2 \%)$ \\
\hline \multirow[t]{6}{*}{ Marital status } & Single & $224(59.9 \%)$ & $77(15.8 \%)$ \\
\hline & Living with a partner & $21(5.6 \%)$ & $70(14.3 \%)$ \\
\hline & Married & $18(4.8 \%)$ & $254(52.1 \%)$ \\
\hline & Separated & $4(1.1 \%)$ & $4(0.8 \%)$ \\
\hline & Divorced & $26(6.9 \%)$ & $77(15.8 \%)$ \\
\hline & Widowed & $81(21.7 \%)$ & $6(1.2 \%)$ \\
\hline \multirow{3}{*}{$\begin{array}{l}\text { Number of strong } \\
\text { COVID-19 } \\
\text { health-related } \\
\text { worries }\end{array}$} & 0 & $253(65.1 \%)$ & $384(80.2 \%)$ \\
\hline & 1 & $79(21.9 \%)$ & $63(13.1 \%)$ \\
\hline & 2 & $47(13 \%)$ & $32(6.7 \%)$ \\
\hline \multirow{3}{*}{$\begin{array}{c}\text { Number of strong } \\
\text { COVID-19 eco- } \\
\text { nomic worries }\end{array}$} & 0 & $270(75.2 \%)$ & $430(90.0 \%)$ \\
\hline & 1 & $59(16.4)$ & $25(5.2 \%)$ \\
\hline & 2 & $30(8.4 \%)$ & $23(4.8 \%)$ \\
\hline
\end{tabular}


Table 3 Prevalence of poor mental health outcomes in clients and staff

\begin{tabular}{|c|c|c|c|c|c|c|c|c|c|}
\hline \multirow[t]{2}{*}{ Outcome } & \multicolumn{3}{|l|}{ Staff } & \multicolumn{3}{|c|}{ Clients } & \multicolumn{3}{|c|}{$\begin{array}{l}\text { Clients excluding } \\
\text { psychiatric hospitals and } \\
\text { nursing homes }\end{array}$} \\
\hline & Total & M & $\mathrm{F}$ & Total & M & $\mathrm{F}$ & Total & M & $\mathrm{F}$ \\
\hline Poor well-being $\%$ & 17.4 & 12.5 & 18.7 & 46.3 & 42.5 & 49.7 & 48.2 & 45.2 & 49.6 \\
\hline Depression, any \% & 22.0 & 14.1 & 24.2 & 58.4 & 55.1 & 61.7 & 58.5 & 53.9 & 60.9 \\
\hline Anxiety, any \% & 14.4 & 10.9 & 15.4 & 44.9 & 42.4 & 47.8 & 43.4 & 37.5 & 46.8 \\
\hline Any outcome, $\%$ & 27.2 & 18.1 & 29.6 & 61.6 & 57.7 & 69.0 & 65.8 & 69.2 & 58.0 \\
\hline
\end{tabular}

Rates of poor mental health outcomes among clients varied across different types of institutions. Highest rates of poor well-being were identified in psychiatric hospitals (61\%), child diagnostic institutes (58\%) and elderly homes (57\%) (Fig. 1). Anxiety was highest in child diagnostic institutes (67\%) followed by residential treatment centres (60\%) and psychiatric hospitals $(60 \%)$. Nearly $80 \%$ of client respondents met criteria for depression in psychiatric hospitals followed by residential treatment centres (74\%) and child diagnostic institutes (67\%). Data from the nursing homes were considered unreliable after they were visually inspected and plotted; we think that this is because of the insufficient cognitive capacity of residents in nursing homes who mostly have a diagnosis of mental retardation among others. To avoid information bias, in further analysis data on clients in nursing homes were excluded.

Highest rates of mental health problems among staff members were also seen in psychiatric hospitals as well as in elderly homes and homes with special arrangements (Fig. 2). In psychiatric hospitals, about $40 \%$ of the respondents met criteria for depression followed by homes with special arrangements (36\%) and elderly homes (20\%). The staff of elderly homes and psychiatric hospitals had the highest levels of anxiety (22\%) followed by homes with special arrangements $(21 \%)$. A similar trend was observed with poor well-being with the highest rates being in psychiatric hospitals and elderly homes (28\%) followed by homes with special arrangements (19\%).

Exclusion of the nursing homes (because of data invalidity - see above) resulted in an increase of prevalence of poor mental health outcomes in clients by 5-10\% (Table 3). However, this was reduced almost to the original estimates after additional removal of
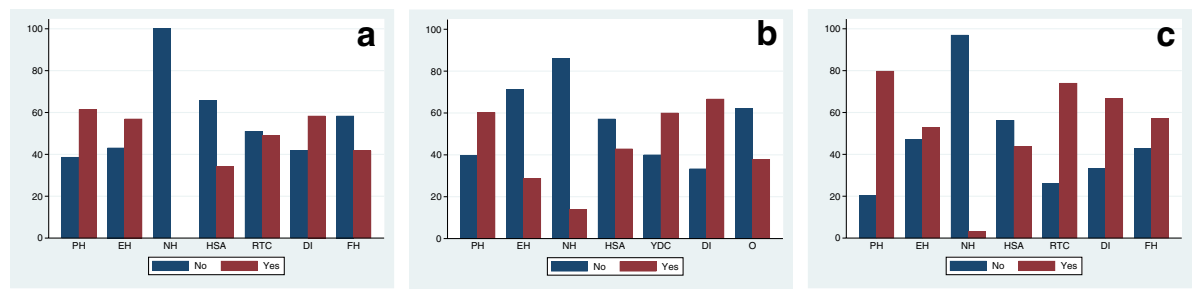

Fig. 1 Prevalence of poor well-being (a), anxiety (b), depression (c) among clients by type of institution PH - Psychiatric hospital EH - Elderly home NH - Nursing Home HSA - Home with special arrangements RTC - Residential treatment centre DI - Child diagnostic institute FH - Foster home. Graphs are made in Stata Software. Figure is made in Stata Software, v.16 

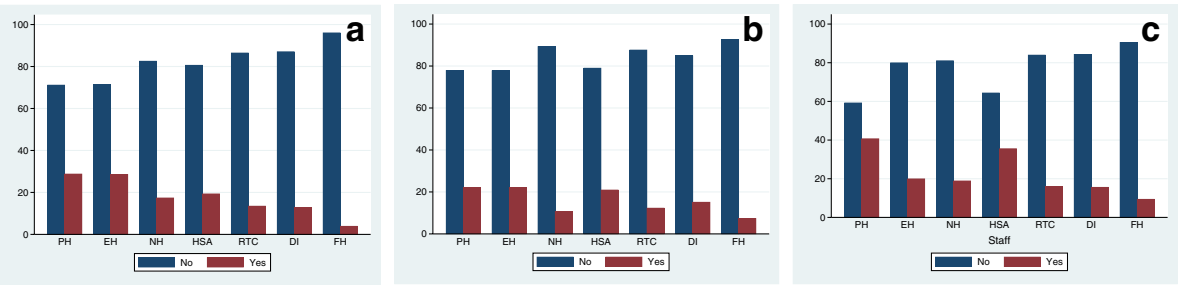

Fig. 2 Prevalence of poor well-being (a), anxiety (b), depression (c) among staff by type of institution PH Psychiatric hospital EH - Elderly home NH - Nursing Home HSA - Home with special arrangements RTC - Residential treatment centre DI - Child diagnostic institute FH - Foster home. Figure is made in Stata Software, v.16

psychiatric hospitals (because of mental health problems being a primary reason for hospitalization). This points to the exceedingly high prevalence of mental health problems in the institutionalized population outside of the psychiatric hospitals.

Table 4 demonstrates that highest rates of anxiety and depression are attributed to the youngest of respondents. As many as $70 \%$ of children below 18 fulfilled criteria for depression, $57 \%$ for anxiety and $50 \%$ for poor well-being. The next most affected is the $70+$ group with more than half of respondents were found to have depression or poor well-being and nearly a third reported symptoms of anxiety.

Most anxiety and depression cases were mild or moderate in both groups (Figs. 3 and Fig. 4). However, when combined, moderate and severe cases of anxiety constituted a prevalence of $26 \%$ in clients and $3.9 \%$ in staff. Moderate and severe depression combined was identified in $40 \%$ of clients and $6.1 \%$ of staff. Overall, $40 \%$ of clients and $7 \%$ of staff had at least one moderate to severe outcome (depression or anxiety). This is reduced to $31.1 \%$ and $6.3 \%$ after exclusion of psychiatric hospitals.

\section{COVID-19 and Mental Health}

The association between COVID-19 related worries and mental health problems has some differences in two respondent groups (Table 5). Overall, there is strong evidence that COVID-19 health-related worries are associated with an increased odds of poor well-being $(2.15,95 \% \mathrm{CI}=1.45 ; 3.19)$, depression $(2.28,95 \% \mathrm{CI}=1.54 ; 3.38)$ and anxiety $(1.92,95$ $\mathrm{CI} \%=1.33 ; 2.77)$ in staff. In clients, these associations are weaker for depression $(1.79$ $95 \% \mathrm{CI}=1.14 ; 2.8)$ and anxiety $(1.6395 \%=1.11 ; 2.4)$, while for well-being there is no statistically significant association. For COVID-19 economic worries in staff, strong evidence of association was only identified with depression $(2.0595 \% \mathrm{CI}=1.33$; 3.17), while

Table 4 Prevalence of poor mental health outcomes in clients stratified by three age groups (psychiatric hospitals and nursing homes excluded)

\begin{tabular}{llll}
\hline Outcome & \multicolumn{2}{l}{ Age group (clients) } \\
\cline { 2 - 4 } & $12-17$ & $18-69$ & $70+$ \\
\hline Poor well-being \% & 50.0 & 35.9 & 54.0 \\
Depression, any \% & 70.2 & 44.7 & 54.0 \\
Anxiety, any \% & 57.3 & 36.6 & 31.5 \\
Any outcome, \% & 72.6 & 57.1 & 63.6 \\
\hline
\end{tabular}



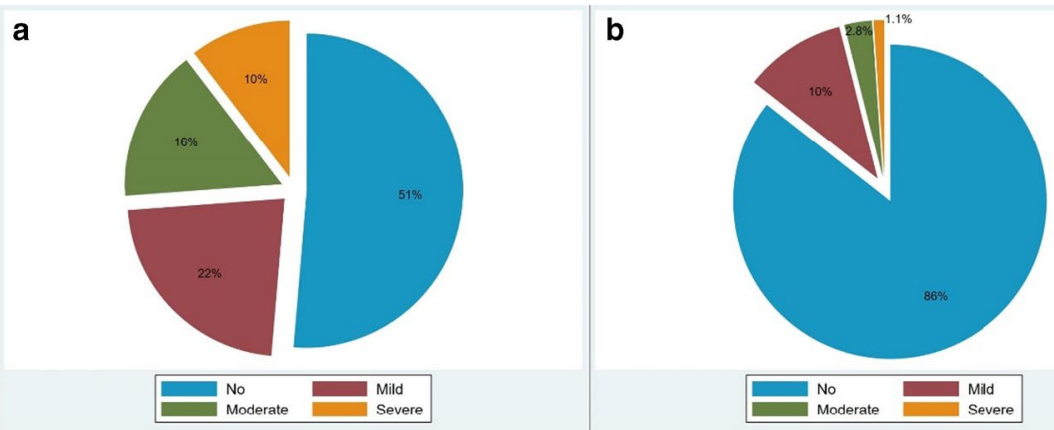

Fig. 3 Anxiety severity in clients (a) and staff (b). Figure is made in Stata Software, v.16

in clients both depression and anxiety were associated with economic worries $(2.2895 \%$ $\mathrm{CI}=1.27 ; 4.08$ and $1.8595 \%$ CI 1.2;2.85 respectively) about the impact of COVID-19 pandemic.

\section{Focus Groups}

\section{Structured Questioning}

All out of 17 adult institutions organized a focus group and reported outcomes for structured questioning, with 16 out of 17 having staff responses and 15 out of 17 having client responses (one institution conducted a client focus group only). For child institutions, 8 out of 10 organized a focus group and reported outcomes for staff and 5 out of 10 provided client focus group responses.

\section{Discrepancies between Participant Groups Responses - Structured Questioning}

In terms of the first theme (Information and communication), in adult facilities, there was an agreement between staff and managers on the lack of information provided by founders or government agencies. Staff and managers also agreed that they provided enough

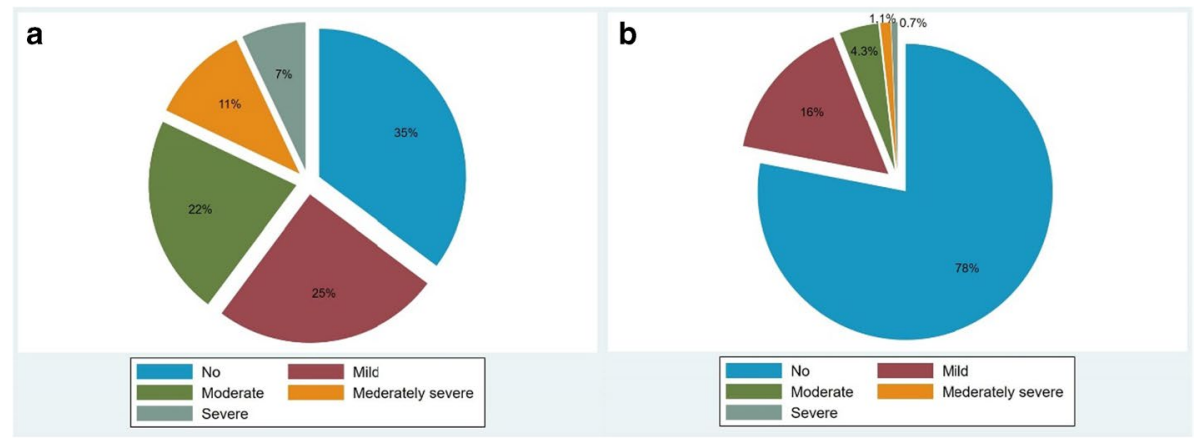

Fig. 4 Depression severity in clients (a) and staff (b). Figure is made in Stata Software, v.16 


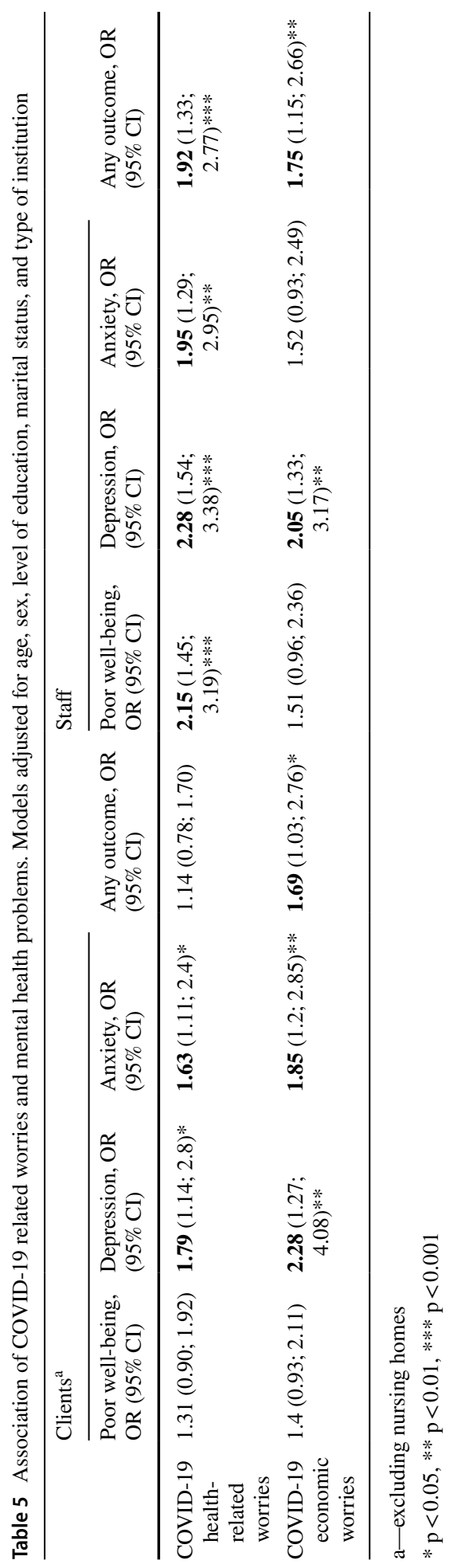


information to clients, which was corroborated by clients' responses. In child institutions, staff and management responses varied slightly on the provision of adequate information, however, similarly to adult institutions provision of information to clients was sufficient and also corroborated by clients' responses.

Theme 2 (Implementation of measures) showed agreement for most structured questions in adult institutions. Staff, managers and clients agreed that they had enough equipment, they were able to provide care to patients with COVID-19 and were regularly tested for the presence of COVID-19. A similar agreement can be seen in child institutions, however, there were some discrepancies where staff and clients reported less social distancing implementation than managers.

For Theme 3 (Delivery of care) there was a slight disagreement between staff and client opinions on the range of services provided, with clients claiming services were more restricted than staff. All groups, however, agreed that physical healthcare was not influenced by the restrictions. Managers and staff agreed admission processes were affected. Finally, all groups also agreed that the free movement of clients was restricted. In child institutions, all groups agreed that both services and physical care were disrupted as well as admissions processes. However, there was disagreement between staff and managers regarding restriction of free movement of clients, more managers disagreed that restriction of movement happened in their institutions than staff.

Finally, in Theme 4 (Quality of experiences), in adult institutions both clients and staff agreed with an increase in anxiety, distress and problematic behaviour while most managers disagreed. Both staff and managers agreed there were new challenges such as increased workload and heightened stress. All groups agreed that they dealt with the situation well. In child institutions, more staff and clients agreed with an increase in mental health issues than managers. More staff also agreed with experiencing new problems than managers. However, similarly to adult institutions, all groups agreed that they were able to overall deal well with the new situation. Details on all discrepancies for every theme can be found in Table 2 in the Online Resource.

\section{Open Questioning}

Table 6 summarizes the number of codes per each analysed category: type of institution, positions and themes. For both adult and child institutions, no differences were observed in reported data between management, staff and clients. Therefore, management and staff results will be merged and will be referred to as staff. Tables 3-10 in the Online Resource show the three most mentioned codes per institution per theme.

\section{Main challenges, Key Best Practices, Key Future Measures - Open Questioning}

\section{Theme 1: Information and Communication}

According to qualitative data, there was a need for the provision of on-time clear information. However, since there was not a singular responsible body, a vast majority of the institutions (both healthcare and social care), faced a lack of clear instructions which would be relevant to their type of institution. 
Table 6 Summary of number of codes generated per institution, position and theme

\begin{tabular}{lll}
\hline Total codes & 1693 & \\
\hline Codes by institution & Adult institutions & 1132 (122 unique values) \\
& Child institutions & 561 (239 unique values) \\
Codes by position & Staff & 1289 (305 unique values) \\
& Clients & 404 (134 unique values) \\
Codes by themes & Theme 1: Information and Communication & A: 298 (19 unique values \\
& Theme 2: Implementation of Measures & C: 153 (59 unique values) \\
& A: 278 (30 unique values) \\
& Theme 3: Delivery of Care & C: 145 (81 unique values) \\
& A: 477 (58 unique values) \\
& Theme 4: Quality of experience & C: 133 (89 unique values) \\
& & A: 79 (31 unique values) \\
& & C: 130 (90 unique values) \\
\hline
\end{tabular}

$A$ Adults, $C$ Children

"Nursing homes have been forgotten in the emergency situation, so we received information from media only." - staff, nursing home.

Those instructions which were sent to institutions were implemented with difficulty and significantly impacted the whole run of the institution. For example, the necessity to create a room for isolation was challenging. Similarly, the obligation to keep distances was not feasible in rooms where beds are closer than 2-m distance. The obligation to wear a facemask the whole day was also seen as a challenge, especially for patients with cognitive decline and children.

Staff closely cooperated with other health and/or social care providers. In that sense, the availability of information and communication technologies (ICT) was appreciated - employees shared information using social networks or intranets. However, there were some challenges with the usage of ICT (such as Wi-Fi not being available in the institution), therefore there was a perceived need to strengthen the ICT area in all institutions.

Another frequently measured best practice was correct communication with clients. Institutions reported using information leaflets, oral communication, but also games and activities (in child institutions). However, correct communication sometimes proved challenging as patients often disregarded social distancing measures, which resulted in staff repeating information frequently.

Securing the availability of clear information in case of another epidemiological situation was identified most frequently as a future measure which needs to be put in place. In general, staff were not provided with timely information regarding the availability of protective equipment, the financial aspects of providing care in different conditions, and the guidelines did not cover specific situations such as a situation when a client has any of the COVID-19 symptoms.

The institutions recommended the coordination of information sharing or the designation of a responsible body who would develop and clearly communicate guidelines to all types of institutions. Similarly, the guidelines and other relevant materials (such as leaflets for patients) should be institution-specific, as there are differences in the 
structure of clients as well as the institution equipment (outdoor spaces, schools in institution premises, etc.)

\section{Theme 2: Implementation of Measures}

At the beginning of the pandemic, there was a severe lack of protective equipment (facemasks, shields). However, this was resolved thanks to the active involvement of the public and staff. Self-help activities were crucial, in helping to overcome the lack of protective equipment. Regarding the other protective measures such as disinfection, and gloves, most institutions reported using up their stock very quickly. This meant that often institutions had to rely on receiving supplies from municipalities and/or the government. There was a lack of optimal coordination regarding the process of supply. Institutions often mentioned that the supplies came late, in some cases, they also mention the quality of the supplies was not adequate.

"Protective equipment was not secured by the establisher [MYES] for a minimum of 2-3 weeks"-staff, foster home.

Social distancing was another measure which had to be undertaken. In some institutions this proved to be easy by denoting recommended distances on floors, creating wards or isolation spaces. The latter was created by closing down wards and sending a proportion of the patients back home. Other institutions created more outdoor spaces. However, some measures were still considered redundant or unfeasible in some facilities.

"Physical distances may not be followed in corridors and rooms. Paradoxically, daily activities were limited, and distances were kept out of a building, but we sleep $50 \mathrm{~cm}$ next to each other, perhaps even less, and we sit side by side in the dining room, or in the smoking-room."- client, psychiatric hospital.

Finally, regular symptom control was an often-mentioned practice. However, institutions were unsure what would be the next step should a patient show symptom of infection. Furthermore, some institutions remained open and kept admitting patients, in this case, most put in place a new system of admissions which would require less contact and screening for COVID-19.

\section{Theme 3: Delivery of Care}

Concerning the service provision, the main challenge was to keep-up daily regimes of clients/patients. On a personal level, there was a reported feeling of uncertainty and fear in all positions (staff - clients). Additionally, communication with patients and their families proved to be an increased burden for staff in child institutions where frequent explaining was necessary. This was also the case in adult institutions with patients with lowered cognitive capabilities. Secondly, a key challenge was a lack of staff. This was due to the occurrence of mandatory quarantines of some employees and/or employees living with a nursing benefit.

A key best practice identified in all institutions was a good attitude of staff, such as the ability of staff to cooperate, share information with clients and willingness to provide extra care to clients. Some institutions also reported employees staying in the institution as a form of quarantine and protection. The importance of active management when resolving 
the measures was reported as important by staff - managers were active in getting the upto-date information, sharing the best practices with other service providers, providing the staff with clear instructions.

Lack of staff during the state-of-emergency was partially solved by the involvement of students and volunteers. However, a member of staff did report that there is a necessity to provide incoming students with basic training, which may be quite time-consuming. In that sense, it may be beneficial to create a list of long-term volunteers in each institution.

"The staff was strengthened by students of medical schools-they helped on walks and shifts, they communicated with patients."- staff, nursing home.

Based on the first experiences with the COVID-19 epidemic, the institutions identified the revision of existing measures as a key future measure. Mainly those related to admissions and discharges of clients. The limited competence in making new admissions and discharges has severely impacted the financial situations of institutions, but also significantly influenced the service provision and compliance to the recommended guidelines. Additionally, there were some mandatory measures which were seen as redundant - such as the obligation to disinfect institution premises even if there was no confirmed infection in the institution or wearing masks indoors.

\section{Theme 4: Quality of Experience}

As the main challenge, institutions often mentioned facing high levels of stress caused by messages in the media (this was mentioned both in the position of staff and clients). While they often used media as a source of information, they also noted that this created undue tension.

A specific main challenge for child institutions was an increase in client's escapes, which then resulted in increases in clients who had to be quarantined. Institutions therefore often debated the implementation of restrictive measures. Moreover, child institutions also saw an increase in aggressive behaviour towards staff and self-harm incidence.

Based on the recommendations from the ministries, daily activities (such as - schooling in child institutions or therapies in adult institutions) were cancelled, therefore, alternative programmes for clients were developed by staff.

Standardized crisis management including the development of a crisis plan for each institution was seen as a crucial future measure. The standardized crisis management would cover the system of information supply to institutions, guidelines on how to treat infected clients or employees and how to secure supplies for an institution (protective equipment, disinfection, tests, medication, food, etc.). Some representatives of residential institutions preferred establishing national crisis management, others would rather prioritize crisis management at a regional level.

\section{Discussion}

The study demonstrates that about $60 \%$ of clients and a fifth of staff in long-stay institutions, who participated in the study, fulfilled criteria for either anxiety, depression or poor mental well-being in the time of enhanced restrictive measures during COVID-19 pandemic. Because of the cross-sectional design of the study and non-existence of baseline assessment, it is impossible to state to which extent poor mental health outcomes 
were caused by the pandemic and related restrictive measures. Results obtained from another longitudinal study, which measured the mental health of a representative sample of the community-dwelling Czech population during the pandemic and retrospectively collected pre-pandemic data showed a rapid increase of moderate to severe depression and anxiety rates after state-of-emergency was announced following by a steady decline over time corresponding to the gradual lifting of the restrictions [19]. This study used a validated questionnaire based on PHQ- 8 and GAD-7 and its results can be compared with our data. It showed that prevalence of moderate to severe depression or anxiety in the general adult population was in the range between $15 \%$ and $11 \%$ in the period when our survey was conducted, which is higher than $6.3 \%$ in staff in our study. Relatively low prevalence of moderate or severe mental health problems in staff is likely explained by secured employment and hence less economic worries compared to the general population, for whom economic difficulties during the pandemic showed to be a very significant factor for worse mental health outcomes [19]. Another reason likely lies in the fact that it is young people (aged 18-24) who were shown to be the most affected, while staff in our study was largely represented by an older age group. Even if psychiatric hospitals are not considered, $31 \%$ of clients were found to have moderate and severe mental health problems. These alarming results warrant appropriate measures and should precede larger studies and implementation research as well as policy revision, particularly with regard to child institutions, since children were shown to be the most vulnerable in our study.

We found that individuals who expressed health or economic worries about the impact of COVID-19 pandemic had significantly higher odds of having at least one mental health problem in staff and depression and anxiety in clients. This is in line with the results of the national Czech survey of the general adult population published recently [21]. These results should be treated with caution since people who previously had symptoms of mental disorders or poor well-being for other reasons not related to the pandemic could be more likely to express worries about COVID-19 situation, therefore further longitudinal investigations are warranted.

\section{Best Practices}

Ultimately, the following best practices were identified as the most viable based on the included institutions' reports. Firstly, a good level of cooperation within the institution, this means good communication of information between staff and patients. Good cooperation is also recommended outside the institution such as with other stakeholders- ministries, outpatient care, hygiene stations, municipalities and social care services. Secondly, establishing systems of information sharing (intranet, social networks) within each institution. Thirdly, putting measures in place to protect and support staff. Institutions in the current study protected staff by dividing them into work teams or created appropriate accommodation spaces for staff. Fourthly, and lastly, establishing a crisis team within each institution.

\section{Needs on the Institution Level}

The data from the included institutions highlighted several needs. Firstly, there is a need for each institution to have a prepared crisis plan which would outline processes and methodologies regarding staff quarantines and patients'/clients' isolation, as well as processes of admission. Perhaps most importantly, such a plan should include detailed staff education (such as correct use of the equipment and how to attend to patients suspected of 
infection). Secondly, there is a need for better information and communication technologies which would allow for smooth communication both within and outside the institution. Thirdly, a significant proportion of institutions would benefit from the involvement of students or volunteers to combat staff shortage. However, it must be noted, such volunteers would need appropriate training. Fourthly, to assure mental health needs are met patients'/clients' regimes should be maintained, this will require alternative activities such as online psychotherapy, education, sports, games and entertainment, phone contact with families but also maintaining contact with external staff (such as psychologists, teachers). Finally, to maintain staff mental health needs regular psychological supervision should be maintained.

\section{Needs on the National Level}

Several needs at the national level emerged. Firstly, there is a need for a crisis management team at the national or regional level, which would act as one responsible body and would therefore provide the necessary guidelines. Secondly, a single system of information sharing should be established which would provide up to date and timely information. This information would not only be about the correct processes regarding the provision of care but also for example how to communicate with patients (an example of this would be materials such as educational leaflets or videos). Thirdly, any information or measure should also be adapted to the specifics of institutions. Fourthly, there is a need for a revision of the current measures when it comes to outdoor activities, school attendance (in the case of child institutions) and visits. Fifthly, perhaps most importantly for child institutions, an adjustment in legislation regarding the use of restrictive measures was demanded from the institutions, this is specifically related to when a client escapes and has to be readmitted. Finally, a revision of the current financing of institutions in time of emergency is required, institutions often call for less bureaucracy when paying for immediate necessary expenses such as protective equipment.

\section{Strengths and Limitations}

The use of quantitative and qualitative approaches allowed us to consider in detail the impact of COVID-19 on the work of long-stay institutions and the mental health of clients and staff of these facilities. The quantitative part provided novel preliminary evidence on the high prevalence of mental health issues in the institutionalized population and its potential relationship to COVID-19 worries, while the qualitative data allowed us to investigate in-depth participants' experiences and understand potential reasons for high anxiety and depression as well as suggestions for improvement during difficult times. Our study benefited from the inclusion of a heterogeneous sample of institutions including both child and adult facilities. In addition to psychiatric hospitals, which are often exclusively the subject of deinstitutionalization debate, six other types of longstay institutions were included in the study, thus providing a large range of experiences and insights from different contexts. Data collection for the study was conducted when the most severe restrictions were imposed by the government, enabling us to obtain unique and valuable data on the experiences and challenges in long-stay institutions. This will allow better help to be offered during the ongoing substantial increase of COVID-19 cases and in the case of further waves or other similar epidemiological situations. To our knowledge, this is the first study that evaluates the well-being and mental 
health of clients and staff of long-stay institutions. This is an area that is often overlooked in global mental health yet is an important topic in historically institutionalized Eastern and Central Europe.

Our study has several limitations. Due to time constraints, we employed a convenience rather than a random sample, which is subject to selection bias and results from our study cannot be generalizable to other long-stay institutions. Additionally, the study does not allow assessing causality of risk factors and outcomes due to its cross-sectional design. All questionnaires used in the study are self-reported tools, which are prone to information bias. Response bias also remains a key limitation for the qualitative data collected in the current study. Focus groups discussions could have been impacted by opinions of more vocal participants, or even participants in a higher position in the case of staff participants. Another limitation to qualitative data is that only transcripts rather than records from focus groups notes were available for analysis, which could have led to some loss of data but enabled the participants of focus groups to feel safer and more open about their responses. Finally, the questions posed in the focus groups had primarily a negative valence-meaning that participants may have felt being asked to only report the negatives.

\section{Conclusion}

The results and the implications of the study came in the time of the substantial surge of COVID-19 cases in the country, which has led to re-introduction of the state-of-emergency and restrictive measures. Alarmingly high prevalence of poor mental health outcomes in clients emphasizes the importance of careful attention to the long-stay institutions during challenging pandemics and measures to be taken to support and promote mental health in these facilities, considering the complexities, as well as the best practices found in this study. Future rigorous research is needed to assess mental health in long-stay institutions both in and out of the context of COVID-19.

Supplementary Information The online version contains supplementary material available at https://doi. org/10.1007/s11126-021-09912-z.

Acknowledgements We would like to thank the data collectors and the study participants for devoting their time to complete the study. We also thank Hana Tomášková for assisting with analysis and translation of the study.

Funding The study was supported by the project 'Sustainability for the National Institute of Mental Health' with a financial support from the Ministry of Education, Youth and Sports of the Czech Republic under Grant LO161 and by the European Union's Horizon 2020 research and innovation programme under grant agreement No 101016233 (PERISCOPE).

Data Availability The data that support the findings of this study are available from the corresponding author, AA, upon request.

\section{Declarations}

Ethical Approval The study was approved by the Ethics Committee of the National Institute of Mental Health.

Informed Consent Written informed consent was obtained from all participants.

Conflict of Interest The authors declare that they have no conflict of interest. 


\section{References}

1. Moesmann Madsen M, Dines D, Hieronymus F. Optimizing psychiatric care during the COVID-19 pandemic. Acta Psychiatr Scand. 2020;142(1):70-1.

2. Li L. Challenges and priorities in responding to COVID-19 in inpatient psychiatry. Psychiatr Serv. 2020;71(6):624-6.

3. Druss BG. Addressing the COVID-19 pandemic in populations with serious mental illness. JAMA Psychiat. 2020;77(9):891-2.

4. Safta-Zecheria L. Challenges posed by COVID-19 to the health of people with disabilities living in residential care facilities in Romania. Disabil Soc. 2020;35(5):837-43.

5. Hsu ST, Chou LS, Chou FH, et al. Challenge and strategies of infection control in psychiatric hospitals during biological disasters-From SARS to COVID-19 in Taiwan. Asian J Psychiatr. 2020;54:102270.

6. Du N, OuYang Y, Chen Y. The experience of prevention measures taken by the psychiatric hospital during the emergence of asymptomatic patients with COVID-19. Psychiatry Res. 2020;291:113109.

7. Thome J, Coogan AN, Fischer M, Tucha O, Faltraco F. Challenges for mental health services during the 2020 COVID-19 outbreak in Germany. Psychiatry Clin Neurosci. 2020;74(7):407.

8. D’Agostino A, Demartini B, Cavallotti S, Gambini O. Mental health services in Italy during the COVID-19 outbreak. Lancet Psychiatry. 2020;7(5):385-7.

9. Chevance A, Gourion D, Hoertel N, Llorca P-M, Thomas P, Bocher R, Moro M-R, Laprévote V, Benyamina A, Fossati P. Ensuring mental health care during the SARS-CoV-2 epidemic in France: A narrative review. L'encephale. 2020;46(3):193-201.

10. Kopelovich SL, Monroe-DeVita M, Buck BE, et al. Community Mental Health Care Delivery During the COVID-19 Pandemic: Practical Strategies for Improving Care for People with Serious Mental Illness. Community Ment Health J. 2021;57(3):405-415.

11. Fegert JM, Schulze UM. COVID-19 and its impact on child and adolescent psychiatry-a German and personal perspective. Ir J Psychol Med. 2020;37(3):243-5.

12. Molodynski A, McLellan A, Craig T, Bhugra D. What does COVID mean for UK mental health care? Int J Soc Psychiatry. 2020;20764020932592. https://doi.org/10.1177/0020764020932592.

13. $80 \%$ of people living with mental illness say current crisis has made their mental health worse. Rethink Mental Illness. 2020. https://www.rethink.org/news-and-stories/news/2020/04/80-of-people-livin gwith-mental-illness-say-current-crisis-has-made-their-mental-health-worse/. Accessed 22 Mar 2021.

14. Brooks SK, Webster RK, Smith LE, Woodland L, Wessely S, Greenberg N, Rubin GJ. The psychological impact of quarantine and how to reduce it: rapid review of the evidence. Lancet. 2020;395(10227):912-20.

15. Long-stay mental health care institutions and the COVID-19 crisis: identifying and addressing the challenges for better response and preparedness. Copenhagen: WHO Regional Office for Europe; 2020. Licence: CC BY-NC-SA 3.0 IGO.

16. Spitzer RL, Kroenke K, Williams JB, Löwe B. A brief measure for assessing generalized anxiety disorder: the GAD-7. Arch Intern Med. 2006;166(10):1092-7.

17. Kroenke K, Spitzer RL, Williams JB. The PHQ-15: validity of a new measure for evaluating the severity of somatic symptoms. Psychosom Med. 2002;64(2):258-66.

18. Topp CW, Østergaard SD, Søndergaard S, Bech P. The WHO-5 well-being index: a systematic review of the literature. Psychother Psychosom. 2015;84(3):167-76.

19. Bartoš V, Cahlíková J, Bauer M, Chytilová J. The impact of the coronavirus pandemic on mental health. [Dopady pandemie koronaviru na duševní zdraví]. Praha: Národohospodářský ústav AV ČR; 2020.

20. Strauss A. Corbin J. Grounded theory methodology: an overview. In: Denzin NK, Lincoln YS, editors. Handbook of qualitative research. Thousand Oaks: Sage Publications; 1994. p. 273-285.

21. Winkler P, Formanek T, Mlada K, et al. Increase in prevalence of current mental disorders in the context of COVID-19: analysis of repeated nationwide cross-sectional surveys. Epidemiol Psychiatr Sci. 2020;29:e173.

Publisher's Note Springer Nature remains neutral with regard to jurisdictional claims in published maps and institutional affiliations. 
Zoe Guerrero is a researcher at the National Institute of Mental Health, Czech Republic (NIMH). She is also the internal evaluator for the Destigmatization project 'Na Rovinu' at the NIMH. She received her master's degree in Global Mental Health from King's College London and the London School of Hygiene and Tropical Medicine. She has worked on research related to cultural psychology, the impacts of mental health stigma and the development of anti-stigma interventions.

Akmal-Alikhan Aliev is a researcher at the Department of Public Mental Health at the National Institute of Mental Health in Klecany, Czech Republic and the WHO Collaborating Centre for Public Mental Health and Service Development. He obtained his first degree in General Medicine at the Tashkent Medical Academy in Uzbekistan. He graduated with a Master of Science degree in Global Mental Health from King's College London and London School of Hygiene and Tropical Medicine. His main interests are deinstitutionalization, development of mental health care and mental health reforms in Eastern Europe and Central Asia.

Lucie Kondrátová is the leader of the Mental health services research workgroup in the National Institute of Mental Health, Czech Republic (NIMH). After obtaining her Master 's Degree in Sociology at the Charles University in Prague, she has been actively involved in studies concerning the socioeconomic impact of mental health services in the Czech Republic. Currently, she is also the scientific coordinator in a psychiatric care reform project focused on the implementation of early detection and early intervention services in the Czech Republic.

Bibiána Jozefiaková obtained her master's degree in Psychology at the University of Presov, Slovakia. Currently, she is a Ph.D. student at Olomouc University Social Health Institute, and her dissertation topic is posttraumatic growth. She also works as the president of the Slovak association of students and graduates of psychology. Her current research interests include the psychotraumatology, psychopathology, and stigma of mental health.

Natálie Nesázalová is a student of Psychology at the Palacky University in Olomouc, Czech Republic. She is undergoing her internship at the Department of Public Mental Health at the National Institute of Mental Health

Josephine Gabriela Saňáková is a student of Andragogy and Sociology at the Palacky University in Olomouc, Czech Republic. She is undergoing her internship at the Department of Public Mental Health at the National Institute of Mental Health.

Petr Winkler obtained his PhD in Health Service and Population Research from the Institute of Psychiatry, Psychology and Neuroscience, King's College London. In 2015 he established the Department of Public Mental Health in the National Institute of Mental Health, Czech Republic and he has been leading the department since then. As of February 2021, the department has been designated as the WHO Collaborating Centre for Mental Health Service Research and Development, and PW became its director. In the recent decade, PW has been involved in the development and implementation of the mental health care reform in the Czech Republic, including leading three nation-wide projects focused on destigmatisation, early interventions in psychosis, and system for evidence-based mental health care development. He co-authored both, National Mental Health Action Plan 2030 and National Suicide Prevention Plan of the Czech Republic. His research is very much focused on improving mental health care in the region of central and eastern Europe. $\mathrm{He}$ is also serving as a Lancet Commissioner for Mental Health Stigma and Discrimination (commission due in 2022). 